\title{
The Picosecond Laser Effects on Tattoo Removal and Metabolic Pathways
}

\author{
Yan $Q u^{\prime}$ \\ Xien Feng ${ }^{2}$ \\ Jin'ning Liang ${ }^{2}$ \\ Junru $\mathrm{Liu}^{3}$ \\ Dong $\mathrm{Gao}^{2}$ \\ 'Department of Dermatology, The \\ Affiliated Hospital of Weifang Medical \\ University, Weifang, Shandong, 261031, \\ People's Republic of China; ${ }^{2}$ Department \\ of Dermatology, Yantai Yuhuangding \\ Hospital, Yantai, 264003, Shandong, \\ People's Republic of China; ${ }^{3}$ Department \\ of Dermatology, Yantai Yuhuangding \\ Hospital Laishan Branch, Yantai, 264003, \\ Shandong, People's Republic of China
}

Correspondence: Dong Gao Email gaodong_yhd@163.com
Objective: We aimed to investigate picosecond laser effects regarding rat tattoo removals. We systematically detected the metabolic pathways considering tattoo pigment particles in rat models.

Materials and Methods: We employed fluorescein to mark the tattoo dye and utilized a pattern needle to prepare tattoo on rat so as to establish the tattoo removal effects of picosecond laser methods employing animal models. We applied a picosecond laser to process the tattoo and observed the effects along with metabolic pathways for tattoo removal via pathology and imaging approaches.

Results: Based on the results of characterization, pathology and fluorescence, we discovered that the picosecond laser could effectively remove the pigment particles on rat skin, part of which would be metabolized out of the body through the circulatory system. After picosecond laser treatment, the fluorescence intensity of the rat skin engraved part was gradually weakened. At $8 \mathrm{~h}$ after the treatment, the metabolic organs had weak fluorescence, and there was no fluorescence in the metabolic organs at $12 \mathrm{~h}$ and $24 \mathrm{~h}$ after the treatment.

Conclusion: The picosecond laser had perfect tattoo removal effects, which could be utilized as a reference for clinical tattoo removal.

Keywords: tattoo, picosecond laser, pattern engraving, dye

\section{Introduction}

Tattoo is a conventional custom, which remains popular in many areas all over the world. However, for various reasons, tattoos would lead to side effects and complications. Infection is a common complication of tattoos. Although most tattoos utilize aseptic techniques, infections may occur due to improper preparation of the tattoo site, incomplete disinfection of equipment, contamination of dyes or products, or improper care after the tattoo or careless personal hygiene. Tumors might also be induced by implantation of undesirable dyes. How to securely and effectively remove tattoos has become an increasing demand among the tattoo population. Currently, many hospitals lack unified operating specifications and standards for tattoo removal technologies. People applied electrocautery, freezing, peeling grinding and skin grafting to remove tattoos, though the effect was not very satisfactory. With the emergence of laser technology, in the 1960s, Laub et al employed ruby laser to treat human tattoos, ${ }^{1}$ which gradually initiated the usage of various laser techniques to treat tattoos. Q-switched laser treatment of tattoos has gradually become the gold standard. ${ }^{2}$ Although the selective thermal effect of Q-switched laser has good advantages in breaking pigments and reducing skin damage, there are still unsolved issues, such as long treatment intervals, multiple 
treatments, residual tattoo pigments and contours, and scars after treatment. ${ }^{3}$ Q-switched Nd-YAG $1064 \mathrm{~nm}$ laser was based upon the theory of selective photothermal effect, and only melanin and black pigment were its target, while it was unable to remove professional tattoo dyes, such as red and green. ${ }^{4}$

Picosecond laser was gradually being applied by clinical staff to remove tattoos. ${ }^{5}$ Nevertheless, due to the lack of relevant theoretical and data support, the removal effect was not good in real application processes. Skin discoloration and other phenomena frequently occur, which made patients suffer. ${ }^{6}$ Clinical issues such as how the dye was metabolized, whether it would cause secondary damage to the human body, etc., remained unknown. ${ }^{7-14}$ The current study utilized rat tattoo model to observe the effect of picosecond laser on tattoo removal.

\section{Materials and Methods SD Rat Models}

One hundred SD male rats $^{5}$ weighing 160 190 g were purchased from Jinan Pengyue Experimental Animal Breeding Co., Ltd. [SCXK (LU)20140007]. We bred SD rats from Wistar rats with albino coat, which were widely employed in pharmacology, toxicology, efficacy and GLP experiments. Breeding and experiments were carried out in the barrier environment facility of Yantai Langdi Biotechnology Co., Ltd. [SYXK (LU)20170028]. Ethics Committee at Shanghai Skin Disease Hospital, Tongji University School of Medicine approved the investigations (approval no. Idswanimall). Guidelines for the ethical review of laboratory animal welfare ${ }^{15}$ were followed for the welfare and treatment of the laboratory animals.

\section{Preparation of Rat Tattoo Model}

After the SD rats were adaptively reared for $1 \mathrm{w}$, we depilated them on the back with a depilatory agent. The area was $3 \times 3 \mathrm{~cm}^{2}$. After being wiped and disinfected with alcohol, we pierced tattoo dye marked with CY-5 leveraging $0.5 \mathrm{~mm}$ pattern needle until it was bare. The engraved area is $1 \times 1 \mathrm{~cm}^{2}$, and the depth is $0.5 \mathrm{~mm}$. Our team fed tattooed SD rats normally in the dark. After $15 \mathrm{~d}$, the inflammatory reaction disappeared and the pigment was stabilized. We performed the first tattoo removal with picosecond laser, and the second tattoo removal was performed on the 30 $\mathrm{d}$ after tattoo. The treatment was delivered with a 1064-nm neodymium-doped yttrium aluminum garnet (Nd: YAG) picosecond laser (PicoWay, Syneron Candela) with the zoom 1064-nm handpiece, spot size $3 \mathrm{~mm}$, and fluence 2.8 $\sim 4.0 \mathrm{~J} / \mathrm{cm}^{2}$. The experiments were done with chloral hydrate and $\mathrm{Cy}-5$ labeled tattoo dye.

\section{Characterization Observation}

During the experiments, we observed inflammatory reaction, scab, pigment, and blistering. We also detected redness and color fading of the tattoo site after picosecond laser treatment. The effects of picosecond laser on the pigment removal from the surface were detected.

\section{In vivo Fluorescence Observation}

$0 \mathrm{~h}, 0.5 \mathrm{~h}, 1 \mathrm{~h}, 2 \mathrm{~h}, 4 \mathrm{~h}, 8 \mathrm{~h}, 12 \mathrm{~h}, 24 \mathrm{~h}$ after the first picosecond laser, we randomly selected $3 \mathrm{SD}$ rats from each group at each slot, which were put into the animal in vivo fluorescence imaging system utilizing $650 \mathrm{~nm}$ laser excitation with $670 \mathrm{~nm}$ reception. We detected the distribution and disappearance of fluorescence in rats at different time points and determined the path of dye metabolism.

\section{Fluorescence Detections of Metabolic Organs}

At the same time points to in vivo fluorescence observations, 3 SD rats were randomly sacrificed and their liver, kidney, intestine, skin, etc., were taken out. Afterwards, we placed the harvested organs in the in vivo fluorescence imaging system using $650 \mathrm{~nm}$ laser excitation with $670 \mathrm{~nm}$ reception. We detected the fluorescence intensity in each organ at different time points so as to further judge the dye metabolism path.

\section{Histological Detections}

After the tattoo pigment was stabilized after picosecond laser treatments, we randomly sacrificed $3 \mathrm{SD}$ rats from each group at each time point. The skin tissue from the tattoo was obtained, part of which was fixed with $10 \%$ neutral formalin solution. We performed conventional dehydration, paraffin embedding, sectioning, and $\mathrm{HE}$ staining to observe the changes in the tissue structure as well as pigment dyes at the tattoo under an optical microscope. The sampling times were: $15 \mathrm{~d}$ after the tattoo, $0 \mathrm{~h}$; $12 \mathrm{~h} ; 15 \mathrm{~d}$ after the first picosecond laser treatment and 15 $\mathrm{d}$ after the second picosecond laser treatment.

\section{Laser Confocal Microscopic Detection}

At the above time points, we extracted the skin tissues with tattoos. The tissues were frozen and sectioned under a laser confocal microscope. We detected the changes of 
the fluorescence that carried by the dye in the tissue such as dispersion and disappearance. The technical parameters that included were excitation wavelength $-650 \mathrm{~nm}$, emission wavelength $-670 \mathrm{~nm}$, laser intensity $9 \%, 650$ V. Sampling time points were: $15 \mathrm{~d}$ after tattooing; $0 \mathrm{~h}$, $4 \mathrm{~h}, 8 \mathrm{~h}, 12 \mathrm{~h}, 24 \mathrm{~h}, 15 \mathrm{~d}$ after the first picosecond laser treatment; and the corresponding time points after the second picosecond laser treatment.

\section{Statistical Analysis}

SPSS 26.0 statistical software SPSS version 26.0 for Windows (SPSS, Inc., Chicago, IL, USA) was utilized to analyze the data and compare differences between groups. The measurement data were expressed as mean \pm standard deviation (SD), and the count data were expressed in percentage. The area of dye and pigment fading after irradiation with different energy-level lasers were tested through R. After adjusting $P$ values, a pairwise comparison was made. $P<0.05$ was regarded as statistically significant.

\section{Results}

\section{Modeling Results}

Within $3 \mathrm{~d}$ after the engraving, redness and swelling at the engraved skin were obvious. On days 5 and 7 , the engraved area showed scabs. On the $10 \mathrm{~d}$, the scabs fell off, and the tattoo dye stabilized. On the $15 \mathrm{~d}$, the dye color at the engraved site was deep with no more changes (Figure 1A). The pathological section of skin tissue demonstrated that at the position of the middle layer of dermis (Figure 1B), many dye particles aggregated and there were no changes on days 11 and 15 . According to the HE staining results and our previous work, ${ }^{5}$ the dye could be determined and the carved part was stabilized. Therefore, the tattoo model was established successfully.

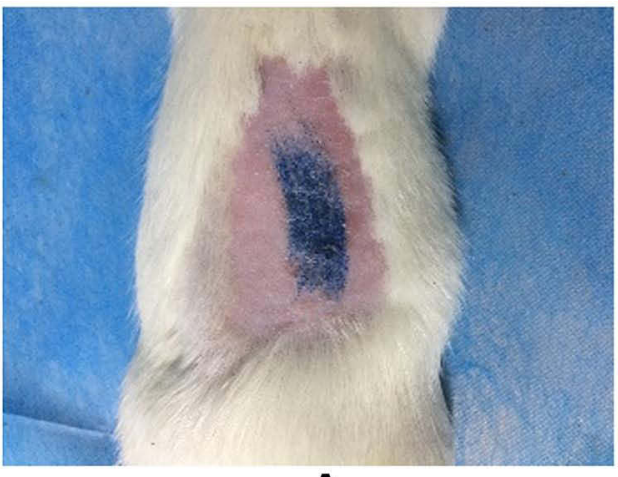

A

\section{General Observation Results}

According to the general observations and our previous work, ${ }^{5}$ the dye state of the rat skin became stable after 15 $\mathrm{d}$ from the dye was engraved. During the subsequent feeding process, the depth of dye on the rat skin was gradually reduced. After about $48 \mathrm{~h}$, it was stabilized. The area of the tattoo was reduced, and the dye appeared scattered with no more flakes. The front became lighter, with about $1 / 2$ of those before treatment. The dye was constantly reduced at a later stage, but the reduction was smaller. After the second picosecond laser, the state of the rat tattoo dye was similar to that of the first time. About $15 \mathrm{~d}$ after the treatment, the rat tattoo basically disappeared. The skin condition at the engraving is shown in Figure 2. The tattoo area was counted as $100 \%$, and the color depth was counted as $100 \%$ $15 \mathrm{~d}$ after engraving. After treatment, the dye fade was calculated, and the status of tattoos at each time point was provided. $15 \mathrm{~d}$ after the first picosecond laser treatment, about $40 \%$ tattoos remained. $15 \mathrm{~d}$ after the second picosecond laser treatment, about $2 \%$ remained.

\section{In vivo Fluorescence Imaging Results}

Through the observation of in vivo fluorescence imaging at different time points, fluorescence was mainly concentrated in the engraved parts $0 \mathrm{~h}$ after the picosecond laser treatment. The fluorescence gradually became stronger in the organs and tail at $0.5 \mathrm{~h} \sim 4 \mathrm{~h}$ after the treatment. The rats were anesthetized at $2 \mathrm{~h}$. Results suggested that different intensities of fluorescence appeared in the intestine, kidney, bladder and other locations (Figure 3A and B). Fluorescence was also found in the rat urines between $2 \mathrm{~h} \sim 4 \mathrm{~h}$. At $8 \mathrm{~h}$, there was almost no fluorescence in the rat indicating that it was metabolized, or the

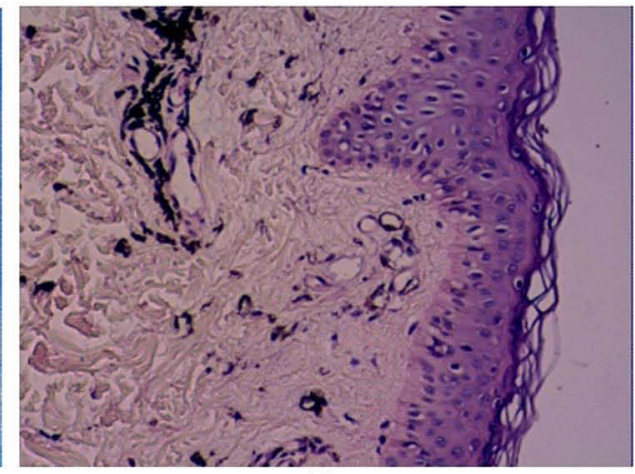

B

Figure I Tattoo dyeing diagram (A) and pathological section (B) on the $15 \mathrm{~d}$. 


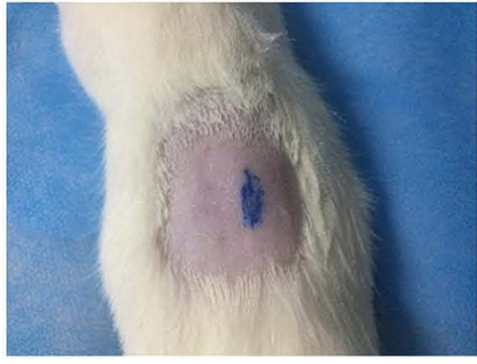

A

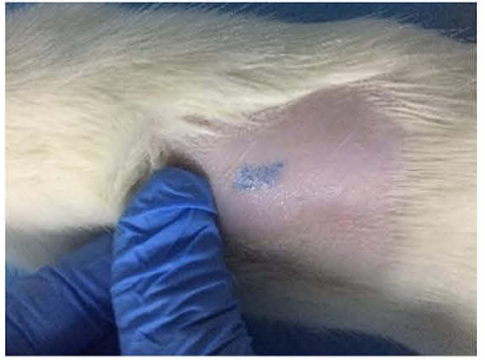

B

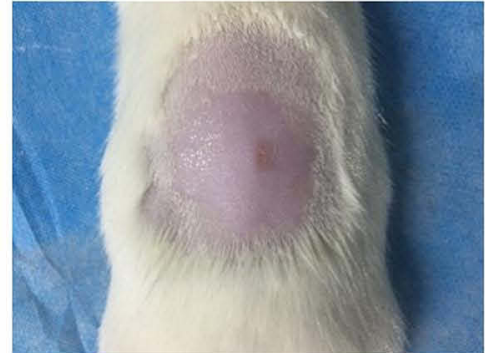

C

Figure 2 General observation results. (A) $10 \mathrm{~d}$ after the first engraving. (B) $15 \mathrm{~d}$ after the first picosecond laser. (C) $15 \mathrm{~d}$ after the second picosecond laser.

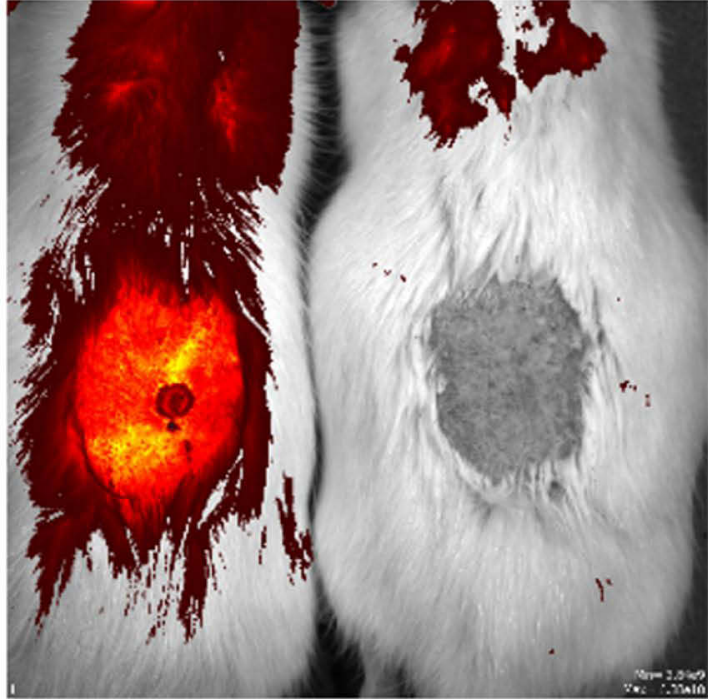

A

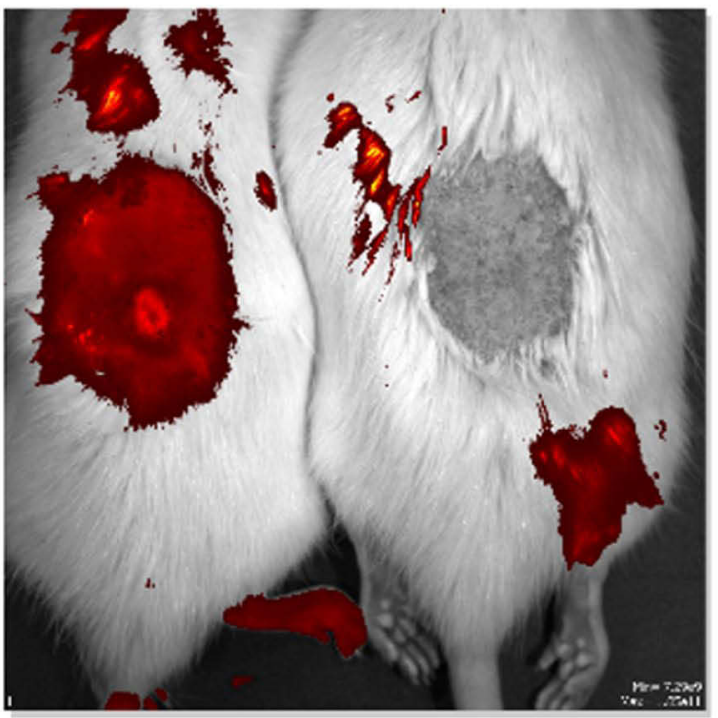

C

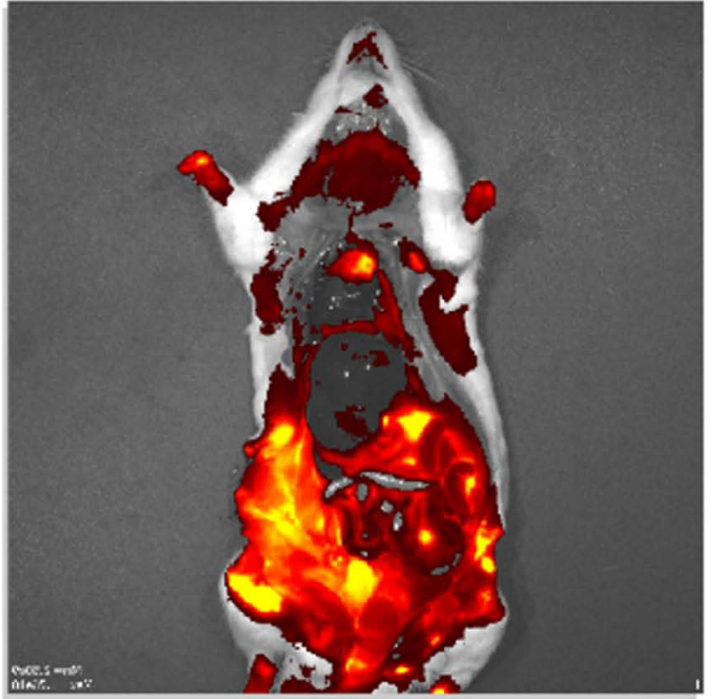

B

Figure 3 (A) Fluorescence imaging results at $2 \mathrm{~h}$ (Normal rat on the right, tattooed rat on the left). (B) Fluorescence imaging results after $2 \mathrm{~h}$ anatomy. (C) Fluorescence imaging results at $12 \mathrm{~h}$ (Normal rat on the right, tattooed rat on the left). 
fluorescence was quenched. No fluorescence was detected in the rat at $12 \mathrm{~h}$ and $24 \mathrm{~h}$ (Figure 3C).

\section{Observation Results of Fluorescence Intensity of Metabolic Organs}

In order to eliminate the interference of the rat's own tissue fluorescence, the rats were anesthetized and sacrificed after the detection of each in vivo fluorescence imaging time point. The metabolic organs were removed, and all metabolic organs were tested in the in vivo fluorescence imaging system. At $0 \mathrm{~h}$, there was no fluorescence in the metabolic organs. At $0.5 \mathrm{~h}$, fluorescence was found in the intestines, liver, and other organs. At $2 \mathrm{~h}$, fluorescence was found in the liver, intestines, kidneys, bladder and other organs, and the fluorescence intensity was strong (Figure 4A). At $4 \mathrm{~h}$, the fluorescence intensity of the liver, intestine, kidney, bladder, etc., was weaker than that of the samples collected at $2 \mathrm{~h}$ (Figure 4B). In the urine samples collected between the two time points, weaker fluorescence was found. At $8 \mathrm{~h}$, the metabolic organs had weak fluorescence (Figure 5). There was no fluorescence in the metabolic organs at $12 \mathrm{~h}$ and $24 \mathrm{~h}$ (Figure 4C).

\section{Histological Experiment Results}

From the results of the skin tissue slices after two picosecond laser treatments, the picosecond laser caused a large number of "cavities" in the deep layer of the skin. They were mainly concentrated between the epidermis and the dermis, and some were located in the dermis. From the Masson staining pictures at each time point, the dye in the skin tissue was in a state of aggregation and clusters $15 \mathrm{~d}$ after the tattoo. It was discovered that the dye particles were broken into tiny particles by laser $0 \mathrm{~h}$ and
$12 \mathrm{~h}$ after the first picosecond laser treatment, and the particle numbers gradually decreased over time. By 15 $\mathrm{d}$ after laser treatment, the residual amount of dye particles was about $1 / 2$ of that before the treatment, and the dye disappeared during the process. In the tissue section, we found that there were macrophages and other cells near the laser channel. From $0 \mathrm{~h}$ to $15 \mathrm{~d}$ after the second picosecond laser treatment, the dye particles continued to metabolize and disappear, and finally, there was almost no particulate dye in the tissue (Figure 5).

\section{Laser Confocal Fluorescence Results}

Laser confocal observation of the fluorescence intensity of skin tissue at the tattoo site revealed that before laser picosecond laser treatment, the fluorescence emitted by the CY5 labeled dye after excitation was concentrated on the skin tissue, and the intensity was high (Figure 6A). The average fluorescence per unit area was 28,965. After the first picosecond laser treatment, the fluorescence in the tissue section at $0 \mathrm{~h}$ was dispersed, and the intensity was slightly weaker than that before the treatment. At $4 \mathrm{~h}$, the fluorescence dispersion state was obvious, and the fluorescence intensity was significantly weakened. The areas were about $80 \%$ of that before the treatment and the average fluorescence intensity per unit area is 21,560 (Figure 6B). Data at $8 \mathrm{~h}, 12 \mathrm{~h}, 24 \mathrm{~h}$ illustrated that the fluorescence intensity was further weakened, and the fluorescence area gradually shrank. The data on the $15 \mathrm{~d}$ after treatment verified that the fluorescence was scattered throughout the tissue. In the fluorescent area, the overall fluorescence intensity was about 43\% (average fluorescence intensity per unit area was 12,432) of that before the treatment (Figure 6C). On the $15 \mathrm{~d}$ after

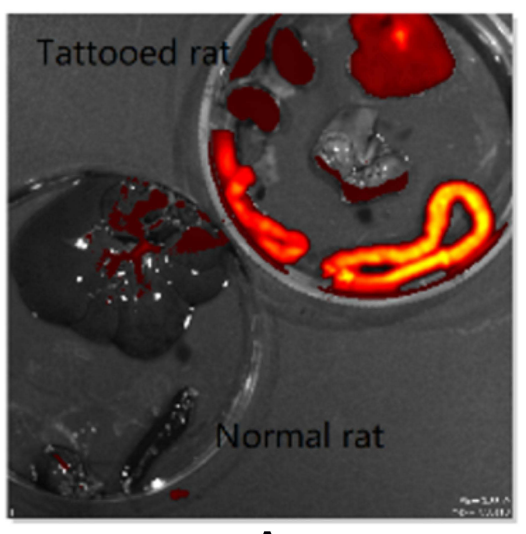

A

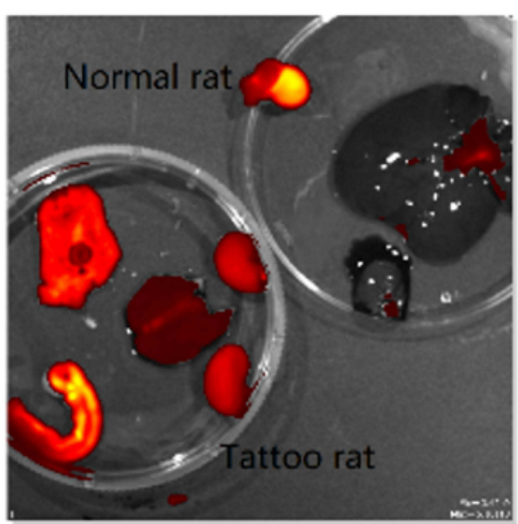

B

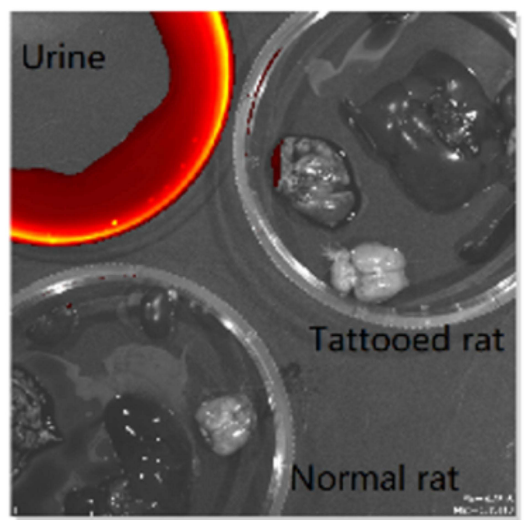

C

Figure 4 Observation of fluorescence intensity of metabolic organ (A) 2 h. (B) 4 h. (C) 12 h. 
A

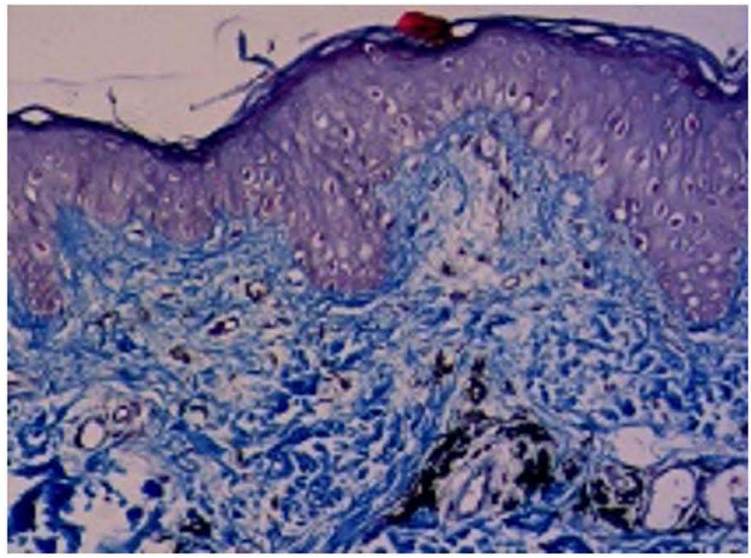

C

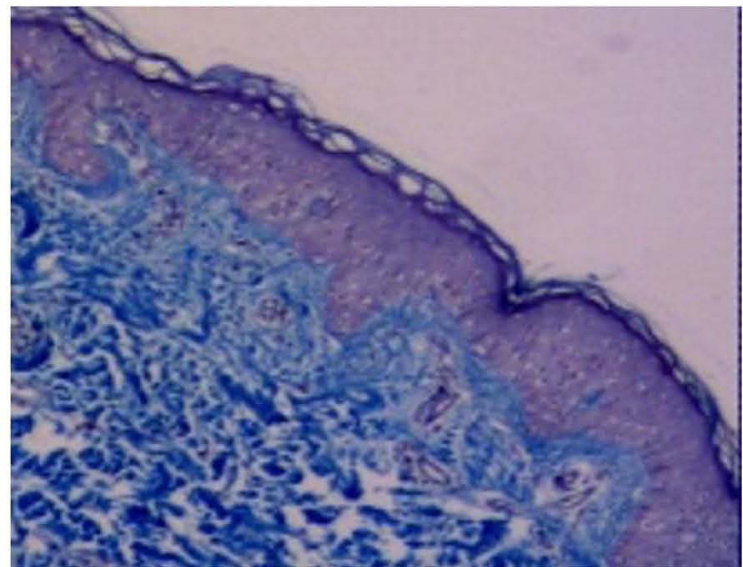

B

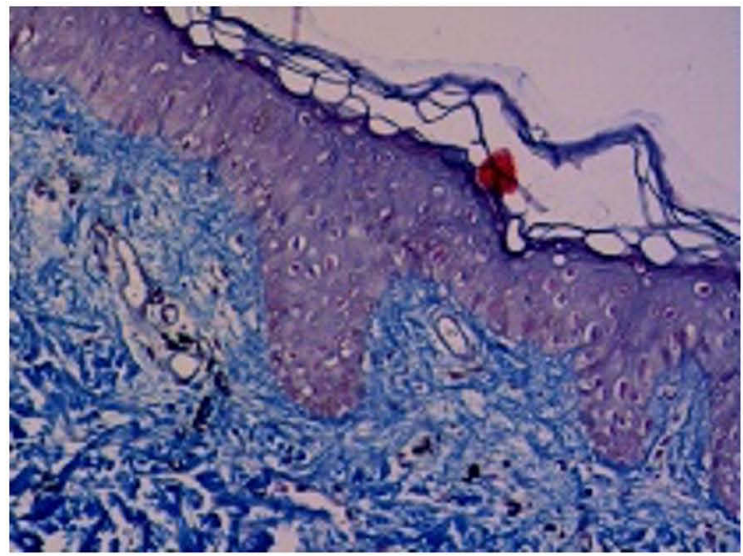

D

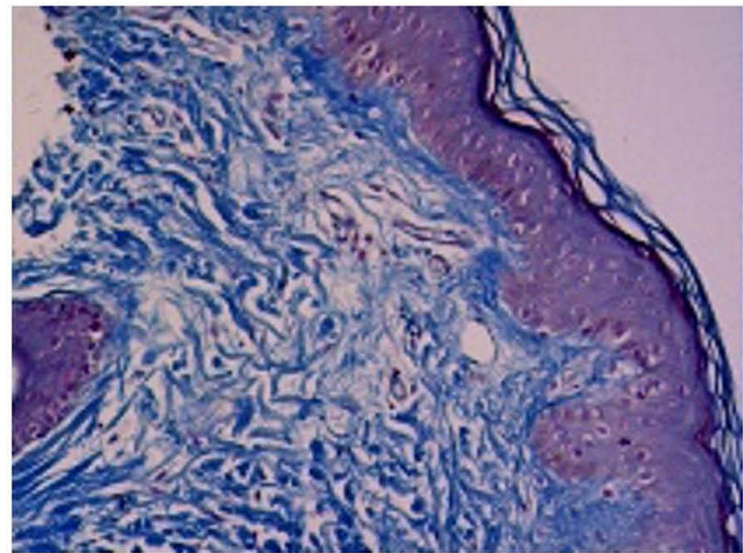

Figure 5 Masson staining results. (A) $15 \mathrm{~d}$ after the tattoo. (B) $12 \mathrm{~h}$ after the first picosecond laser treatment. (C) $15 \mathrm{~d}$ after the first picosecond laser treatment. (D) 15 $\mathrm{d}$ after the second picosecond laser treatment.

the second picosecond laser treatment, the average fluorescence intensity per unit area was only 126 (Figure 6D).

\section{Discussions}

Picosecond laser has great advantages in treating benign pigment diseases. Since the pulse width of the picosecond laser is about $10 \sim 100$ times shorter than that of the traditional short pulse (nanosecond) laser, the high peak wave of this ultrashort pulse laser can produce a strong photomechanical effect on the body instead of traditional nanosecond laser. Because of its photothermal effect, picosecond laser is more effective in the treatments of

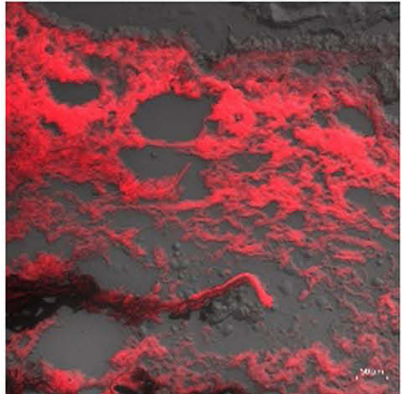

A

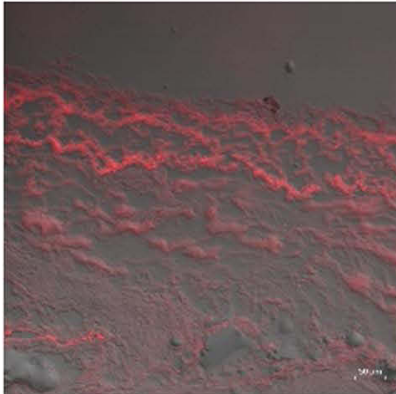

B

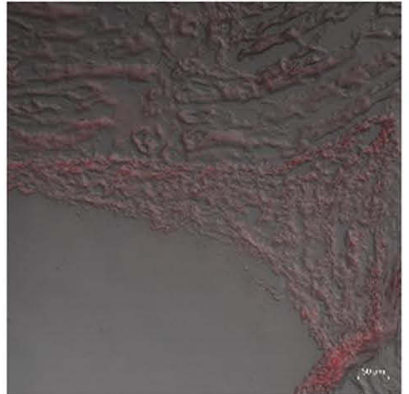

C

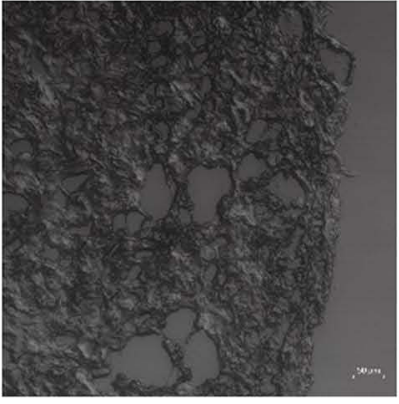

D

Figure 6 Laser confocal fluorescence observation results. (A) Before picosecond laser treatment. (B) $4 \mathrm{~h}$ after the first picosecond laser treatment. (C) $15 \mathrm{~d}$ after the first picosecond laser treatment. (D) $15 \mathrm{~d}$ after the second picosecond laser treatment. 
tattoos as well as other diseases than traditional shortpulse lasers.

As the picosecond laser is a relatively new technology, most of the existing related researches are limited to the clinical observation of laser on pigmented diseases, scars, photoaging and other diseases. ${ }^{16}$ In one study, the control group utilized red tattoo particles. After the shock wave effect of the short pulse Q-switched laser, relatively small black particles were formed. In terms of safety, after PicoWay picosecond laser treatment, it is easier to find complete cell membranes and organelles under the electron microscope. The transmission of the shock wave effect is only for the pigment, and there is not much heat energy to destroy the surrounding tissues. Therefore, it is not applicable in clinical practice. ${ }^{17}$ Under the premise of achieving the same therapeutic effects, the energy of picosecond laser treatment is only $1 / 3$ or even $1 / 4$ of that in Q-switched laser. When the treatment energy is reduced, the comfort of the treatment process is improved, and the safety of the overall treatment is improved as a result. ${ }^{18}$ Compared with the traditional short-pulse nanosecond laser, the ultra-pulse picosecond laser has better absorption of pigments, which greatly reduces the damage to surrounding tissues that is caused by non-specific photothermal effects and has less inflammation as well as lower incidence of staining. ${ }^{19,20}$

Because the size of tattoo particles is between $40 \sim 300$ $\mathrm{nm}$ in the body, the TRT of most tattoo pigments is in the picosecond range. When the pulse width is compressed, the photomechanical effect of picoseconds makes it dependent on selective spectral absorption. For instance, the pulse width of $532 \mathrm{~nm}$ picoseconds matches the particle size of yellow tattoo pigments, so the removal rate for refractory yellow tattoos is also compared. ${ }^{8}$ In terms of safety, picosecond lasers produce less tissue damage and have better removal effects compared to nanosecond lasers. $^{21}$ A single-blind separation random experiment showed that the $1064 / 532 \mathrm{~nm}$ picosecond laser has a removal rate of more than $75 \%$ on tattoo pigments, and its effectiveness is stronger than the $1064 / 532 \mathrm{~nm}$ short pulse Q-switched laser. $^{22}$

From the current study, we concluded that the dye particles were broken into tiny particles by the picosecond laser at the engraved area, which were directly metabolized out of the body through macrophage phagocytosis or other pathways. We discovered that the tattoo dye labeled with CY-5 could stably exist in the skin marking area of rats like ordinary dyes. After picosecond laser treatments, the tattoo dye was broken into tiny particles at the marking position, possibly passing through the tissue part. It entered the circulatory system, which was metabolized out of the body. Two picosecond laser treatments could effectively remove all dyes from the engraved parts, indicating that the picosecond laser had a good effect towards tattoo removal.

In humans, histological evidence indicates that all tattoo particles are found in macrophages, fibroblasts and mast cells around three months post-insertion. While in our investigations, we found that rat skin might process tattoo ink insertion faster than human skin. ${ }^{5}$ Although present research conducted explorations on the effect of picosecond laser on tattoo removal and the metabolic pathways of tattoo dyes, it needs in-depth investigations on the removal mechanism and removal pathways. Subsequent work in the area would be carried out for clinical applications in the mechanism to provide further supports.

\section{Conclusions}

In this study, CY5 fluorescein was employed to label the tattoo dye, and the labeled dye was engraved on the rat skin to realize real-time tracking in rats. The established rat tattoo model was processed by picosecond laser, and fluorescein was excited by the in vivo fluorescence system and laser confocal system. The results demonstrated that after picosecond laser treatment, the fluorescence intensity of the rat skin engraved part was gradually weakened. After two treatments, no fluorescence was detected in the engraved part. At the same time, it was found that after each laser treatment, different metabolic organs will have different intensities of fluorescence at different time points. Some dyes may be directly metabolized out of the body through the skin. Combined with characterization observation results and histological observation results, it was further confirmed that picosecond laser could remove tattoos effectively.

\section{Acknowledgments}

This research was funded by Major Scientific and Technological Innovation Program from the Department of Science and Technology of Shandong Province (grant no. 2019JZZY011103). Authors would like to express their gratitude to editor and anonymous reviewers for the suggestive comments, which helped improve this presentation. 


\section{Disclosure}

The authors report no conflicts of interest in this work.

\section{References}

1. Laub DR, Yules RB, Arras M, et al. Preliminary histopathological observation of Q-switched ruby laser radiation on dermal tattoo pigment in man. J Surg Res. 1968;8(5):220-224. doi:10.1016/00224804(68)90090-5

2. Huerta Rivera G, Lopez Zaldo JB, Arreola Jauregui IE, et al. Clearance of facial tattoo with triple-wavelength picosecond laser and fractionated carbon dioxide laser. Dermatol Ther. 2019;32(1): e12766. doi:10.1111/dth. 12766

3. Khunger N, Molpariya A, Khunger A. Complications of tattoos and tattoo removal: stop and think before you ink. J Cutan Aesthet Surg. 2015;8(1):30-36. doi:10.4103/0974-2077.155072

4. Taro K, Kotaro I, Muneo M. Ultrashort-pulse (picosecond) oscillating laser. Aesthet Dermatol. 2015;25:412-416.

5. Qu Y, Wang L, Zhou PY, Zhang W. Efficient picosecond laser for tattoo removal in rat models. Med Sci Monit. 2020;26:E924583-1-7. doi:10.12659/MSM.924583

6. Freedman JR, Kaufman J, Metelitsa AI, et al. Picosecond lasers: the next generation of short-pulsed lasers. Semin Cutan Med Surg. 2014;33(4):164-168. doi:10.12788/j.sder.0117

7. Alabdulrazzaq H, Brauer JA, Bae YS, et al. Clearance of yellow tattoo ink with a novel $532 \mathrm{~nm}$ picosecond laser. Lasers Surg Med. 2015;47(4):285-288. doi:10.1002/lsm.22354

8. Bernstein EF, Schomacker T, Basilavecchio LD, et al. A novel dual-wavelength, Nd: YAG, picosecond-domain laser safely and effectively removes multicolor tattoos. Lasers Surg Med. 2015;47 (7):542-548. doi:10.1002/1sm.22391

9. Bernstein EF, Bhawalkar J, Schomacker KT. A novel titanium sapphire picosecond-domain laser safely and effectively removes purple, blue, and green tattoo inks. Lasers Surg Med. 2018;50(7):704-710. doi: $10.1002 / 1 \mathrm{sm} .22942$

10. Weiss RA, McDaniel DH, Weiss MA, et al. Safety and efficacy of a novel diffractive lens array using a picosecond $755 \mathrm{~nm}$ alexandrite laser for treatment of wrinkles. Lasers Surg Med. 2017;49(1):40-44. doi:10.1002/1sm.22577

11. Wu DC, Fletcher L, Guiha I, et al. Evaluation of the safety and efficacy of the picosecond alexandrite laser with specialized lens array for treatment of the photoaging decolletage. Lasers Surg Med. 2016;48(2):188-192. doi:10.1002/1sm.22427
12. Dierickx C. Using normal and high pulse coverage with picosecond laser treatment of wrinkles and acne scarring: long term clinical observations. Lasers Surg Med. 2018;50(1):51-55. doi:10.1002/lsm.22763

13. Haimovic A, Brauer JA, Cindy Bae YS, et al. Safety of a picosecond laser with diffractive lens array (DLA) in the treatment of Fitzpatrick skin types IV to VI: a retrospective review. $\mathrm{J} \mathrm{Am} \mathrm{Acad} \mathrm{Dermatol.}$ 2016;74(5):931-936. doi:10.1016/j.jaad.2015.12.010

14. Jang WH, Yoon Y, Kim W, et al. Visualization of laser tattoo removal treatment effects in a mouse model by two-photon microscopy. Biomed Opt Express. 2017;8(8):3735-3748. doi:10.1364/BOE.8.003735

15. MacArthur Clark JA, Sun D. Guidelines for the ethical review of laboratory animal welfare People's Republic of China National Standard GB/T 35892-2018 [issued 6 February 2018 effective from 1 September 2018]. Anim Model Exp Med. 2020;3(1):103-113. doi:10.1002/ame2.12111

16. Artzi O, Mehrabi JN, Koren A, et al. Picosecond 532-nm neodymium-doped yttrium aluminium garnet laser-a novel and promising modality for the treatment of cafe-au-lait macules. Lasers Med Sci. 2018;33(4):693-697. doi:10.1007/s10103-017-2373-9

17. Choi MS, Seo HS, Kim JG, et al. Effects of picosecond laser on the multi-colored tattoo removal using Hartley Guinea pig: a Preliminary Study. PLoS One. 2018;13(9):e0203370. doi:10.1371/journal.pone. 0203370

18. Pinto F, Grosse-Buning S, Karsai S, et al. Neodymium-doped yttrium aluminium garnet (Nd:YAG) 1064-nm picosecond laser vs. Nd:YAG 1064-nm nanosecond laser in tattoo removal: a randomized controlled single-blind clinical trial. Br J Dermatol. 2017;176(2):457-464.

19. Friedman DJ. Successful treatment of a red and black professional tattoo in skin type VI with a picosecond dual-wavelength, neodymium-doped yttrium aluminium garnet laser. Dermatol Surg. 2016;42(9):1121-1123. doi:10.1097/DSS.0000000000000780

20. Guss L, Goldman MP, Wu DC. Picosecond $532 \mathrm{~nm}$ neodymium-doped yttrium aluminium garnet laser for the treatment of solar lentigines in darker skin types: safety and efficacy. Dermatol Surg. 2017;43(3):456-459. doi:10.1097/DSS.0000000000000922

21. Ahn KJ, Zheng Z, Kwon TR, Kim BJ, Lee HS, Cho SB. Pattern analysis of laser-tattoo interactions for picosecond- and nanosecond-domain 1064-nm neodymium-doped yttrium-aluminumgarnet lasers in tissue-mimicking phantom. Sci Rep. 2017;7(1):1533. doi:10.1038/s41598-017-01724-1

22. Lorgeou A, Perrillat Y, Gral N, Lagrange S, Lacour JP, Passeron T. Comparison of two picosecond lasers to a nanosecond laser for treating tattoos: a Prospective Randomized Study on 49 patients. Eur Acad Dermatol Vnereol. 2017;32(2):265. doi:10.1111/jdv.14492
Clinical, Cosmetic and Investigational Dermatology is an international, peer-reviewed, open access, online journal that focuses on the latest clinical and experimental research in all aspects of skin disease and cosmetic interventions. This journal is indexed on CAS.
The manuscript management system is completely online and includes a very quick and fair peer-review system, which is all easy to use. Visit http://www.dovepress.com/testimonials.php to read real quotes from published authors. 